\title{
The inhibition of histone deacetylase 8 suppresses proliferation and inhibits apoptosis in gastric adenocarcinoma
}

\author{
SHIYUAN SONG ${ }^{1 *}$, YING WANG $^{1 *}$, PO XU $^{2 *}$, RUINA YANG $^{1}$, \\ ZHIKUN MA ${ }^{1}$, SHUO LIANG ${ }^{1}$ and GUANGPING ZHANG ${ }^{1}$ \\ Departments of ${ }^{1}$ Oncology, ${ }^{2}$ Urology, The First Affiliated Hospital of Henan University \\ of Science and Technology, Luoyang, Henan 471003, P.R. China
}

Received July 12, 2015; Accepted September 3, 2015

DOI: $10.3892 /$ ijo.2015.3182

\begin{abstract}
Histone deacetylase 8 (HDAC8), a unique member of class I HDACs, shows remarkable correlation with advanced disease stage. The depletion of HDAC8 leads to inhibition of proliferation, apoptosis and cell cycle arrest in multiple malignant tumors. However, little is known about the contribution of HDAC8 to the tumorigenesis of gastric cancer (GC). The present study investigated expression of HDAC8 in GC cell lines and tissues, and the roles of HDAC8 inhibition in the proliferation, cell cycle and apoptosis of gastric cancer cells and explored the potential mechanisms. In the present study, quantitative real-time reverse transcription polymerase chain reaction (qRT-PCR), western blotting, and immunohistochemistry were used to examine the mRNA and protein expression of HDAC8 in GC cell lines and tissues. Then, the correlation between the clinicopathological parameters and the expression of HDAC8 was assessed. Finally, siRNA transfection and HDAC8 plasmid was performed to explore the functions of HDAC8 in GC progression in vitro. We found that the expression of HDAC8 was significantly upregulated both in GC cell lines and tumor tissues compared to human normal gastric epithelial cell, GES-1 and matched non-tumor tissues. Furthermore, depletion of HDAC8 remarkably inhibited GC cell proliferation, increased the apoptosis rate and $\mathrm{G} 0 / \mathrm{G} 1$ phase percentage in vitro. Western blotting showed that the
\end{abstract}

Correspondence to: Dr Ying Wang or Dr Guangping Zhang, Department of Oncology, The First Affiliated Hospital of Henan University of Science and Technology, Luoyang, No. 24 Jinghua Road, Luoyang, Henan 471003, P.R. China

E-mail: yingw_215@163.com

E-mail: 949898789@qq.com

${ }^{*}$ Contributed equally

Abbreviations: HDAC, histone deacetylase; GC, gastric cancer; siRNA, small interfere RNA; qRT-PCR, reverse transcription polymerase chain reaction

Key words: HDAC8, Bmf, apoptosis, oncogene, gastric cancer expression of protein promoting apoptosis such as, Bmf, activated caspase-3, caspase- 6 were elevated following HDAC8 depletion. Our data exhibited an important role of HDAC8 in promoting gastric cancer tumorigenesis and identify this HDAC 8 as a potential therapeutic target for the treatment of gastric cancer.

\section{Introduction}

Gastric adenocarcinoma (GC) is still one of the most common and aggressive carcinomas worldwide, especially in China where it ranks third in incidence and also third in mortality rate in malignant tumors, with 420,489 new cases and 297,496 deaths including 206,704 males and 90,792 females in 2011 (1). In the United States, the estimated new cases and deaths of gastric cancer are 24,590 and 10,720, respectively, in 2015 (2). Hence, the burden of gastric cancer is increasing. However, the mechanisms of the gastric cancer tumorigenesis is still unclear, to explore the molecular mechanism and therefore the potential therapeutic targets is of great importance for gastric cancer patients.

Epigenetic modifications have been found to be involved in the tumorigenesis, development and progression of gastric cancer, such as promoter DNA hypermethylation of tumor suppressors as well as post-translational alterations of histones (3). Previous studies showed that depletion of histone deacetylase is an effective way to inhibit the proliferation, promote cell cycle arrest and induce apoptosis of multiple malignant tumors (4), including breast (5), pancreatic (6), prostate (7), colorectal (8), hepatocarcinoma (9), lung cancer (10), leuchemia (11), gastric cancer (12), gliomas (13), and cervical cancer (14) which suggested that HDACs are the potential therapeutic targets for treatment of cancer.

The histone deacetylase (HDAC) family consists of 18 members (15), which are grouped into four separate classes, class I, HDAC1, HDAC2, HDAC3 and HDAC8; class II, HDAC4, HDAC5, HDAC6, HDAC7, HDAC9 and HDAC10; class IV, HDAC11; and 7 sirtuins $(4,16)$. The class I HDACs (HDAC1, 2, 3 and 8) are most closely related to the yeast (Saccharomyces cerevisiae) transcriptional regulator RPD3. HDACs function as key regulators of chromatin structure and post-translational modifiers of numerous key proteins in any cell type and tissue $(4,17,18)$. Although HDAC1-3 and 
6 were highlighted in most disease-oriented research (19), lately HDAC8 has become increasingly important as a drug target $(20,21)$. HDAC8 is expressed in multiple adult malignant tumor entities, including lung, colon, pancreas and breast as well as in childhood tumor entities such as neuroblastoma (19). Depletion of HDAC8 by small interference RNA (siRNA) transfection inhibits proliferation of human lung, colon, and cervical cancer cell lines, moreover, the forced expression of HDAC8 promotes proliferation and inhibits apoptosis in hepatocellular carcinoma $(22,23)$. It has been reported that HDAC8 mediated regulation of Bcl-2-modifying factor (BMF) via cooperation with STAT3 (24). BMF plays an important role in the execution of apoptosis triggered by the metabolite methylselenopyruvate, which is an inhibitor of HDAC8 (25). However, the expression, function and mechanism of HDAC8 in $\mathrm{GC}$ remain unclear.

In the present study, we found that the expression of HDAC8 was significantly upregulated both in GC cell lines and tumor tissues. HDAC8 knockdown significantly inhibited GC cell proliferation, induced cell cycle arrest and cell apoptosis. Furthermore, forced expression of HDAC8 promoted GC cell proliferation, inhibited cell cycle arrest and cell apoptosis. In addition, suppression of HDAC8 also resulted in the upregulation of BMF transcription followed by increased expression of cleaved caspase- 3 and caspase- 6 .

\section{Materials and methods}

Ethics statement. Written informed consent was obtained from patients before obtaining tissue samples. The procedures used in the present study were approved by the Institutional Review Board of the First Affiliated Hospital, Henan University of Science and Technology and conformed to the Helsinki Declaration and to local legislation.

Patients and samples. GC tissues and their corresponding normal gastric tissues from $51 \mathrm{GC}$ patients treated with surgery in 2008 in our hospital (First Affiliated Hospital, Henan University of Science and Technology, Henan, China) were enrolled in the present study. GC was confirmed by expert histopathologist examination in all these patients. The clinicopathological characteristics of the 57 patients with gastric cancer are shown in Table I.

Cell lines and culture. GC cell lines SGC7901, MKN45, MKN28, BGC823, AGS and GC9811, and the immortalized normal gastric epithelial cell line GES-1 were kindly donated by Professor Daiming Fan (The Fourth Military Medical University) and NCI-N87 were purchased from Shanghai Bioleaf Biotech Co., Ltd. (Shanghai, China). All cell lines were maintained in our institute according to the recommended protocols. Cells were cultured in RPMI-1640 medium (Invitrogen, Carlsbad, CA, USA) supplemented with 10\% fetal bovine serum (FBS; Invitrogen) at $37^{\circ} \mathrm{C}$ in a $5 \% \mathrm{CO}_{2}$ incubator (Thermo Scitific Forma).

Real-time quantitative reverse-transcription ( $q R T-P C R)$. Total RNA was extracted from all the cell lines using TRIzol (Invitrogen) according to the manufacturer's protocol. RNA was stored at $-80^{\circ} \mathrm{C}$ prior to qRT-PCR analysis. HDAC8
Table I. The relationship between the clinical parameters and thHDAC8 (mean \pm SEM) mRNA expression in primary gastric adenocarcinoma.

\begin{tabular}{lccc}
\hline $\begin{array}{l}\text { Clinical } \\
\text { parameters }\end{array}$ & $\mathrm{N}(\%)$ & $\begin{array}{c}\text { Relative expression } \\
(\text { mean } \pm \text { SEM })\end{array}$ & P-value \\
\hline $\begin{array}{l}\text { Age (years) } \\
\geq 60\end{array}$ & $31(60.8)$ & $4.04 \pm 0.27$ & 0.63 \\
$<60$ & $20(39.2)$ & $3.94 \pm 0.19$ & \\
Gender & & & \\
$\quad$ Male & $36(70.6)$ & $3.86 \pm 0.20$ & 0.58 \\
Female & $16(29.4)$ & $4.06 \pm 0.28$ & \\
Size (cm) & & & \\
$\geq 5$ & $29(56.7)$ & $5.20 \pm 0.24$ & $<0.0001$ \\
$<5$ & $22(43.3)$ & $2.42 \pm 0.027$ & \\
Histological & & & \\
differentiation & & & \\
Well & $17(33.3)$ & $2.12 \pm 0.11$ & $<0.0001$ \\
Moderately & $17(33.3)$ & $4.03 \pm 0.23$ & \\
Poorly & $17(33.3)$ & $5.89 \pm 0.29$ & \\
Lymph node & & & $<0.0001$ \\
metastasis & & & \\
No & $14(27.5)$ & $2.08 \pm 0.14$ & \\
Yes & $37(72.5)$ & $4.72 \pm 0.26$ & \\
TNM stage & & & \\
I & $12(23.5)$ & $2.04 \pm 0.14$ & $<0.0001$ \\
II & $18(35.3)$ & $3.84 \pm 0.17$ & \\
III & $21(41.2)$ & $5.32 \pm 0.26$ & \\
\hline
\end{tabular}

expression was detected by PCR amplification of a 210-bp product with the primer pair: forward, 5'-TGCAGCATCTCC AGAAGGTC-3' and reverse, 5'-TCTTTGCATGATGCCC CCT-3'. PCR conditions were: 30 cycles and the temperature $60^{\circ} \mathrm{C}$. GAPDH was used as the internal control. PCR products were separated on a $1.5 \%$ agarose gel stained with ethidium bromide and visualized with UV.

RNA interference. siRNAs and negative control (Shanghai GenePharma Co., Ltd., Shanghai, China) were used to downregulate HDAC8 in BGC-823 and MKN28 cells using Lipofectamine 2000 (Life Technologies) according to the manufacturer's instructions. siRNAs sequences are as follows: siRNA-2 forward, 5'-UUGAGAUAACAAAAACCAGAU-3' and reverse, 5'-CUGGUUUUUGUUAUCUCAAUG-3'; NC siRNA forward, 5'-UUCUCCGAACGUGUCACGUTT-3' and reverse, 5'-ACGUGACACGUUCGGAGAATT-3'.

Cells containing siRNA constructions were named siHDAC8 and siCtrl. Cells containing plasmid-HDAC8 (Guangzhou RiboBio Co., Ltd., Guangzhou, China) and the negative control (NC) (Guangzhou RiboBio) plasmid were named as the cell line-HDAC8 and the cell line-NC, respectively. Cells were plated into 6-well plates and the siHDAC8 and siCtrl were transfected into cells using transfection reagents when cells were $40 \%$ confluent. These cells were used for western blot analysis, qRT-PCR and in vitro experiments. 
MTT assay. Cells were transfected with $100 \mathrm{nM}$ miR-216b inhibitor (Shanghai GenePharma), mimics (Guangzhou RiboBio), or 100 nM siRNA-HDAC8 (Guangzhou RiboBio) as previously described (26), then seeded in 96-well plates ( $2 \times 10^{3} /$ well) $24 \mathrm{~h}$ later. Cell viability was examined by the 3-2,5-diphenyl tetrazolium bromide (MTT) assay (27-29) according to the manufacturer's instructions (Sigma, St. Louis, MO, USA) at designated times.

Colony formation assay. BGC-823 and MKN28 cells with or without HDAC8 knockdown or forced expression were seeded into $100-\mathrm{mm}$ dishes at a density of 5,000 cells/well. After incubation for an additional 7 days, the cells were fixed with methanol for $15 \mathrm{~min}$, and then stained with $0.1 \%$ crystal violet for another $15 \mathrm{~min}$, and colonies of $>50$ cells were manually counted $(30,31)$. The experiments were performed in independent triplicates.

Cell cycle and apoptosis assay. To detect cell cycle and apoptosis alterations, cells were grown in 6-well plates and treated with siHDAC8, siCtrl, HDAC8 or NC for $48 \mathrm{~h}$. For cell cycle analysis, cells were harvested and fixed with ice-cold $75 \%$ ethyl alcohol at $4^{\circ} \mathrm{C}$ overnight and incubated with DNA Prep kit (Beckman Coulter, Fullerton, CA, USA) in the dark for 30-60 min. For apoptosis analysis, cells were harvested, washed twice using phosphate-buffered saline, and fixed in $70 \%$ ethanol at $4^{\circ} \mathrm{C}$ overnight. They were then incubated with propidium iodide at room temperature for $1 \mathrm{~h}$ and analyzed by flow cytometry using a FACScan flow cytometer (BD Biosciences, Mountain View, CA, USA). All the results were from 3 independent experiments.

Western blot analysis. Proteins from GC cell lines and paired fresh GC and tumor-adjacent normal gastric tissues were extracted with RIPA (Beyotime Institute of Biotechnology, Shanghai, China). The following process was carried out as described $(15,32)$. The primary antibodies used for western blot analysis: HDAC8 (1:600, ab18968; Abcam), Bmf (1:500, ab9655; Abcam), caspase-3 (1:500, 710431; Thermo Fisher Scientific), caspase-6 (1:500, MA5-11527; Thermo Fisher Scientific).

Immunohistochemistry. Paraffin sections, 4- $\mu \mathrm{m}$, were baked for $2 \mathrm{~h}$ at $65^{\circ} \mathrm{C}$ and deparaffinized. Antigen retrieval was performed using citrate sodium buffer $(\mathrm{pH} 7.2)$ at $95^{\circ} \mathrm{C}$ for $15 \mathrm{~min}$ and then slides were cooled at room temperature for $30 \mathrm{~min}$. After being treated with $3 \%$ hydrogen peroxide for 15 min to block the endogenous peroxidase, the sections were treated with normal goat serum confining liquid for 30 min to reduce non-specific binding and then rabbit polyclonal anti-HDAC8 (1:200, ab18968; Abcam) was incubated the sections for $12 \mathrm{~h}$ at $4^{\circ} \mathrm{C}$. After re-warming for $1 \mathrm{~h}$ and washing 5 times, sections were incubated with secondary antibody for $30 \mathrm{~min}$ at room temperature. Diaminobenzidine (DAB) was used for color reactions. Subsequent immunohistochemical staining was scored as previously described $(33,34)$.

Statistical analysis. Data were expressed as means \pm standard errors of three independent experiments. For statistical tests,
A
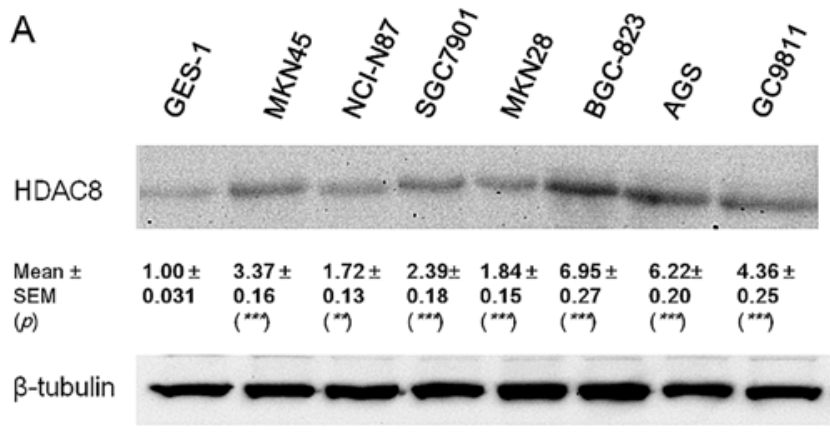

B

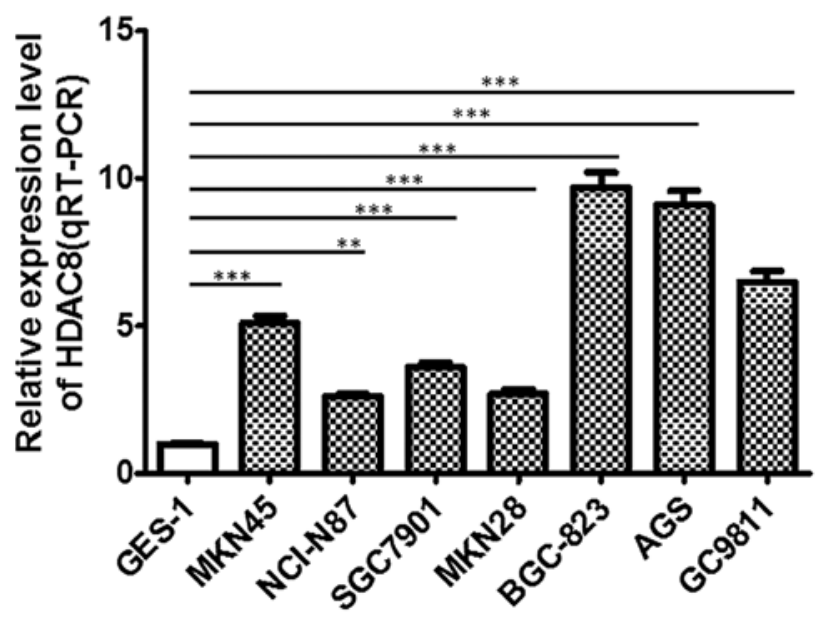

Figure 1. HDAC8 was upregulated in gastric cancer cell lines. HDAC8 expression in seven gastric cancer cell lines and the immortalized normal gastric epithelial cell line GES-1, were quantified by western blotting (A, $\mathrm{n}=3$, paired t-test, ${ }^{* * *} \mathrm{P}<0.0001,{ }^{* *} \mathrm{P}<0.01$ ) and quantitative RT-PCR (qRTPCR) (B, n=3, paired t-test, $\left.{ }^{* * *} \mathrm{P}<0.0001,{ }^{* *} \mathrm{P}<0.001\right)$. Results in $\mathrm{A}$ and $\mathrm{B}$ are representative findings from three or more independent experiments, and all the values are shown as mean \pm SEM, tubulin served as a loading control.

we used the SPSS statistical software package, version 17.0 (SPSS, Inc., Chicago, IL, USA). The Student's t-test and the one-way analysis of variance (ANOVA) were performed to analyze relative band densities in western blotting and MTT optical density values. P-values $<0.05$ were considered statistically significant.

\section{Results}

HDAC8 is upregulated in gastric cancer cell lines. To further examine these findings, using western blotting experiments, we found that HDAC8 protein expression was also upregulated in 7 gastric cancer cell lines compared to GES-1 ( $\mathrm{P}<0.0001)$, and was significantly higher in BGC823, AGS and GC9811 cell lines than in NCI-N87, SGC7901, MKN45 and MKN28 (Fig. 1A). To further investigate the expression pattern of HDAC8 in GC, we then performed qRT-PCR in these gastric cancer cell lines and GES-1. We found that HDAC8 mRNA expression was also significantly upregulated in all the gastric cancer cell lines compared to GES-1 ( $\mathrm{P}<0.0001)$. Moreover, HDAC8 mRNA expression was significantly higher in poorly-differentiated cell lines BGC823, AGS and GC9811 (mean \pm SEM, 8.43 \pm 0.9821 ), compared to other moderately and well-differentiated cell lines, SGC7901, MKN45, MKN28 and BGC823 (mean \pm SEM, 3.5 \pm 0.5788$)(\mathrm{P}=0.0114)$ (Fig. 1B). 


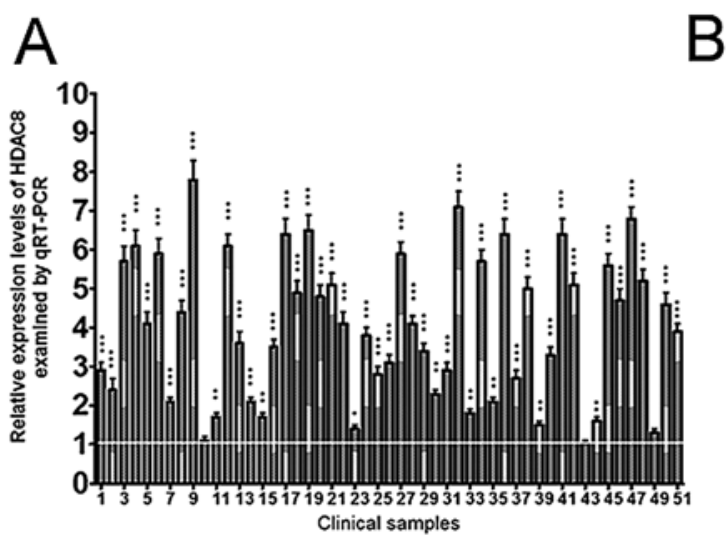

B
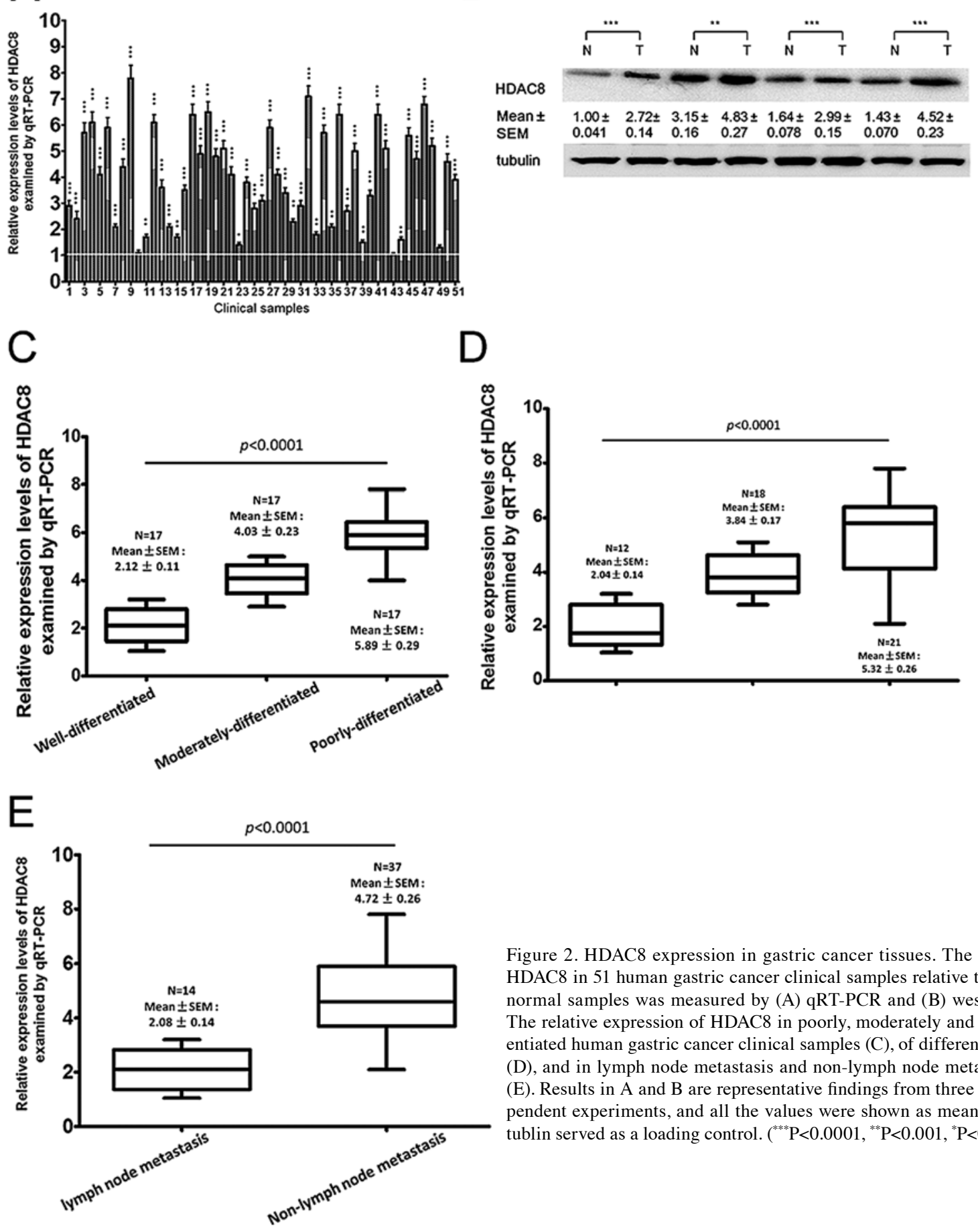

Figure 2. HDAC8 expression in gastric cancer tissues. The expression of HDAC8 in 51 human gastric cancer clinical samples relative to their paired normal samples was measured by (A) qRT-PCR and (B) western blotting. The relative expression of HDAC8 in poorly, moderately and highly differentiated human gastric cancer clinical samples (C), of different TNM stages (D), and in lymph node metastasis and non-lymph node metastasis groups (E). Results in A and B are representative findings from three or more independent experiments, and all the values were shown as mean \pm SEM, $n=3$, tublin served as a loading control. $\left({ }^{* * * *} \mathrm{P}<0.0001,{ }^{* *} \mathrm{P}<0.001,{ }^{*} \mathrm{P}<0.05\right)$.

HDAC8 is upregulated in gastric cancer tissues. To examine HDAC8 expression in GC tissues, 51 cancer tissues, including 17 well, 17 moderately and 17 poorly differentiated tissues and matched non-tumor tissues were used. By qRT-PCR, we found that HDAC8 was significantly upregulated in $47(92.2 \%)$ gastric cancer clinical tissues, compared with non-cancerous tissues (Fig. 2A). Then, HDAC8 expression was examined by immunohistochemistry and western blotting, which indicated that 45 and 44 patients had significantly upregulated HDAC8 expression, as detected by immunohistochemistry (Fig. 2B) and western blot analysis, respectively (Fig. 2C). To gain further insight into this observation, we examined the relationship between HDAC8 expression and the clinical parameters of the patients, and found that HDAC8 expression positively correlated with lymph node metastasis, tumor size, TNM stage and negatively with the histological differentiation (Table I), but did not correlate with age or gender.

Depletion of HDAC8 inhibits proliferation in human gastric cancer cells in vitro. To investigate the role of HDAC8 in the proliferation of human gastric cancer cells, we used BGC823 and MKN28 cells to knockdown and overexpress HDAC8. 
A

A
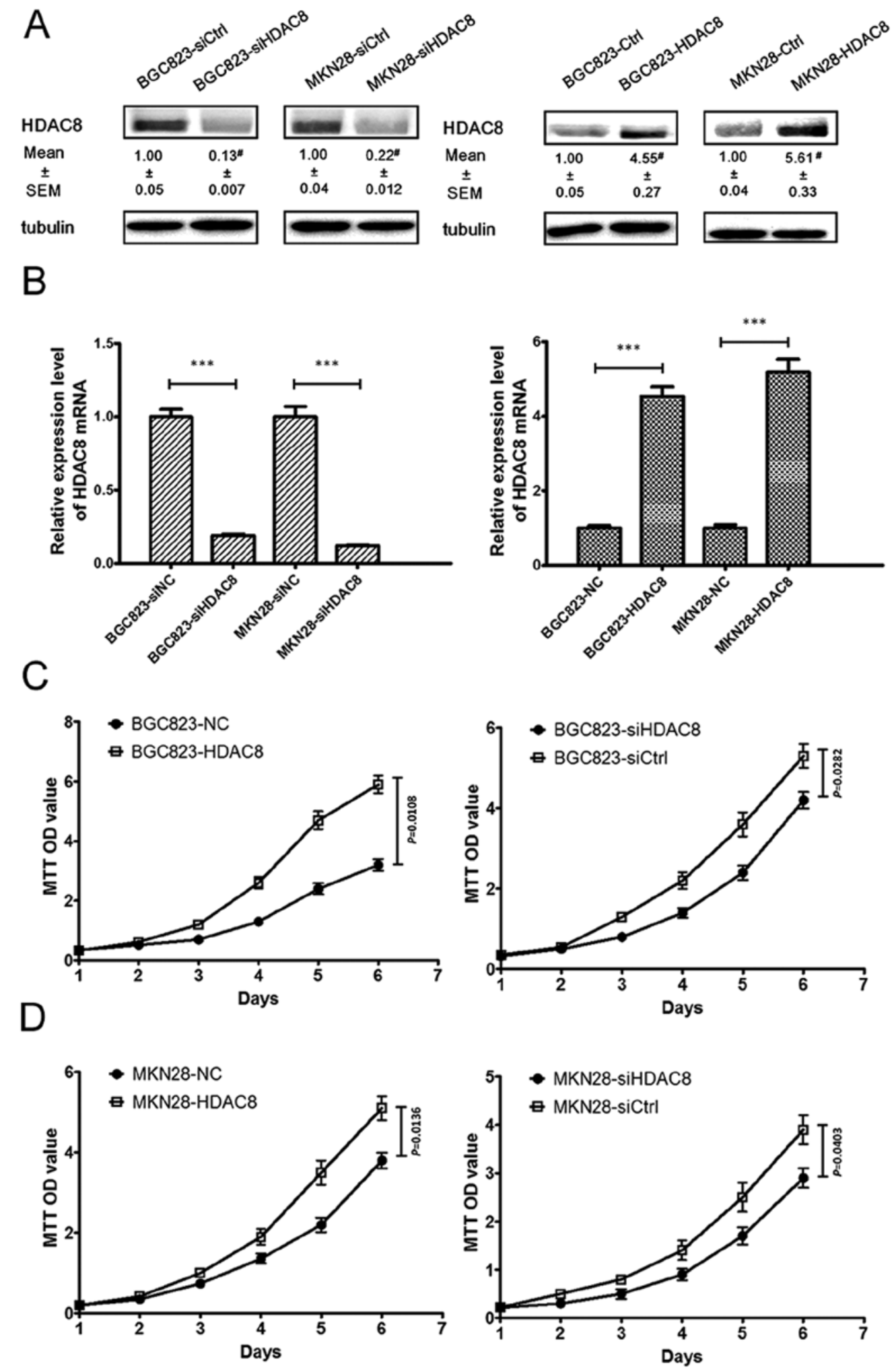

Figure 3. Effect of HDAC8 on proliferation and growth of gastric cancer cell lines BGC-823 and MKN28. (A) Western blotting and (B) qRT-PCR methods were used to examine relative HDAC8 expression. (C and D) The growth of cells over 6 days was examined using MTT assay.

Using siHDAC8 or pcDNA3.1(+)-HDAC8 transfection which was followed with antibiotic selection to gain stable clones, we knocked down or upregulated HDAC8 expression. The expression levels were examined via western blotting (Fig. 3A) and qRT-PCR (Fig. 3B). As shown in Fig. 3C and $\mathrm{D}$, using MTT method, we found that HDAC8 overexpres- sion led to a significant increase in cell proliferation, while HDAC8 knockdown led to a significant decrease in cell proliferation. To further validate the function of HDAC8 in the GC proliferation, we performed plate colony experiments to assess the effect of depletion and upregulation of HDAC8 on the GC cell proliferation. We found that results of the 

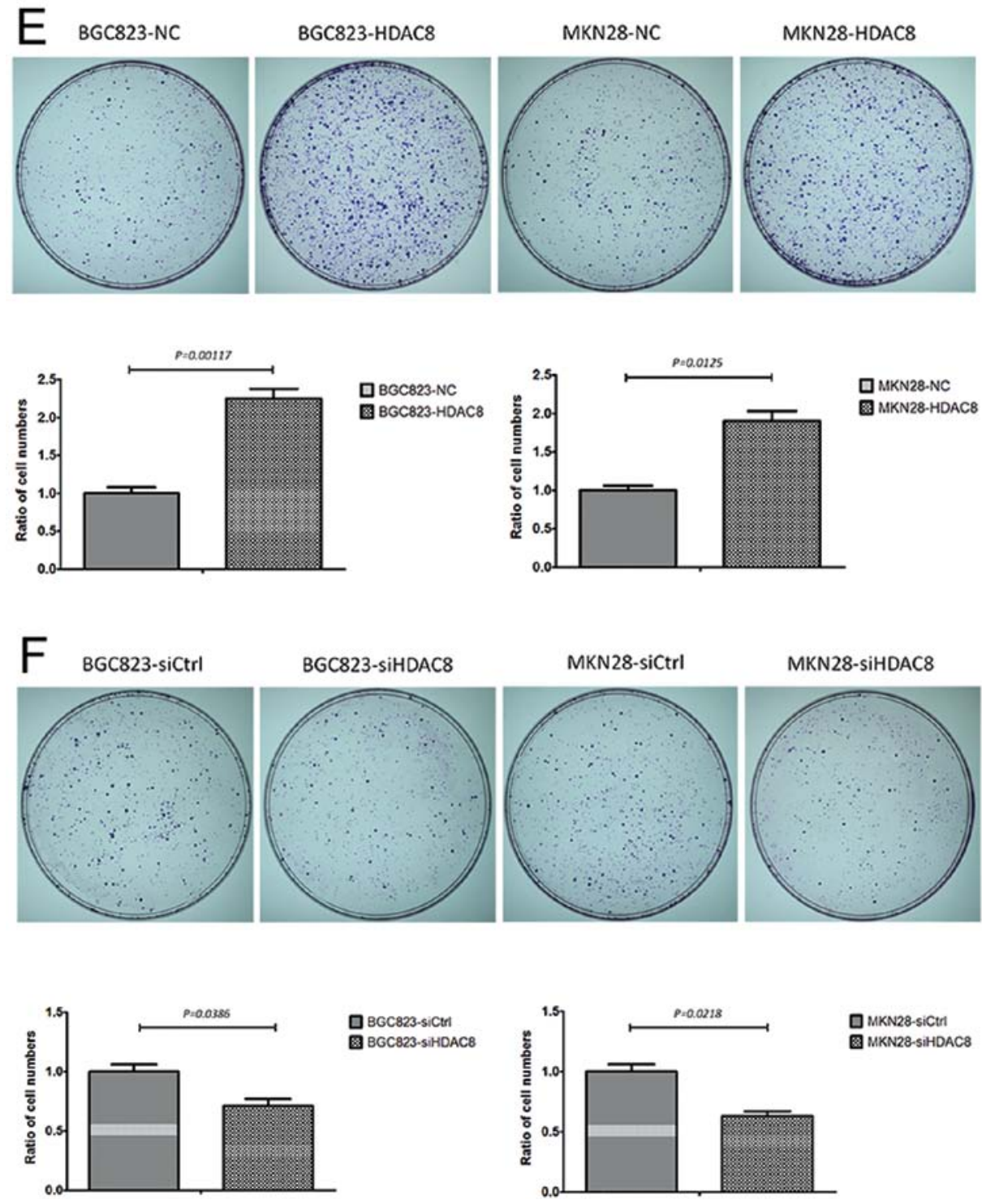

口MKN28-sictr OMKN28-SIHDAC8

Figure 3. Continued. Plate clone experiments of (E) BGC-823 and (F) MKN28 cells were terminated at the 7th day following transfection. Results in A, E and $\mathrm{F}$ are representative findings from three or more independent experiments, and all the values were shown as mean \pm SEM, $\mathrm{n}=3$, tublin served as a loading control. $\left({ }^{* * *} \mathrm{P}<0.0001,{ }^{* *} \mathrm{P}<0.001,{ }^{*} \mathrm{P}<0.05\right)$.

plate colony experiments were in line with that of the MTT assay (Fig. 3E and F).

Knockdown of HDAC8 promoted G0/G1 arrest and apoptosis of gastric cancer. To further study the mechanism by which HDAC8 knockdown or overexpression affected proliferation, cell cycle progression and apoptosis were analyzed using flow cytometry. The MKN28-siHDAC8 cells showed a delayed, while MKN28-HDAC8 exhibited a shortened G1 phase compared with corresponding control groups (Fig. 4A). Moreover, we also found that the apoptotic rates increased significantly in the MKN28-siHDAC8 group after siHDAC8 transfection was performed for $48 \mathrm{~h}$, compared to the control group (Fig. 4B).

Suppression of the HDAC8-induced upregulation of proteins involved Bmf-mediated apoptosis. Previous studies showed that the HDAC8 inhibitor MSP triggers Bmf-mediated apoptosis independent of p21 induction via inducing of pro-apoptotic
BMF expression (24). To investigate the apoptotic mechanism by which siHDAC8 induced apoptosis in more detail, several factors that are pro-apoptotic or indicative apoptotic were examined. In the present study, we first assessed Bmf, activated caspase-3, and activated caspase- 6 using western blotting and found that Bmf, activated caspase-3, and activated caspase- 6 were significantly upregulated when HDAC8 was downregulated (Fig. 5A). The qRT-PCR results were in line with that of western blotting (Fig. 5B).

\section{Discussion}

Epigenetics is the study of heritable alterations in gene expression that are not accompanied by the corresponding change in DNA sequence. There are three interlinked epigenetic processes which regulate gene expression at the level of chromatin, that is, DNA methylation, nucleosomal remodeling and histone covalent modifications. Post-translational modifications that occur on certain amino acid residues of the tails of 

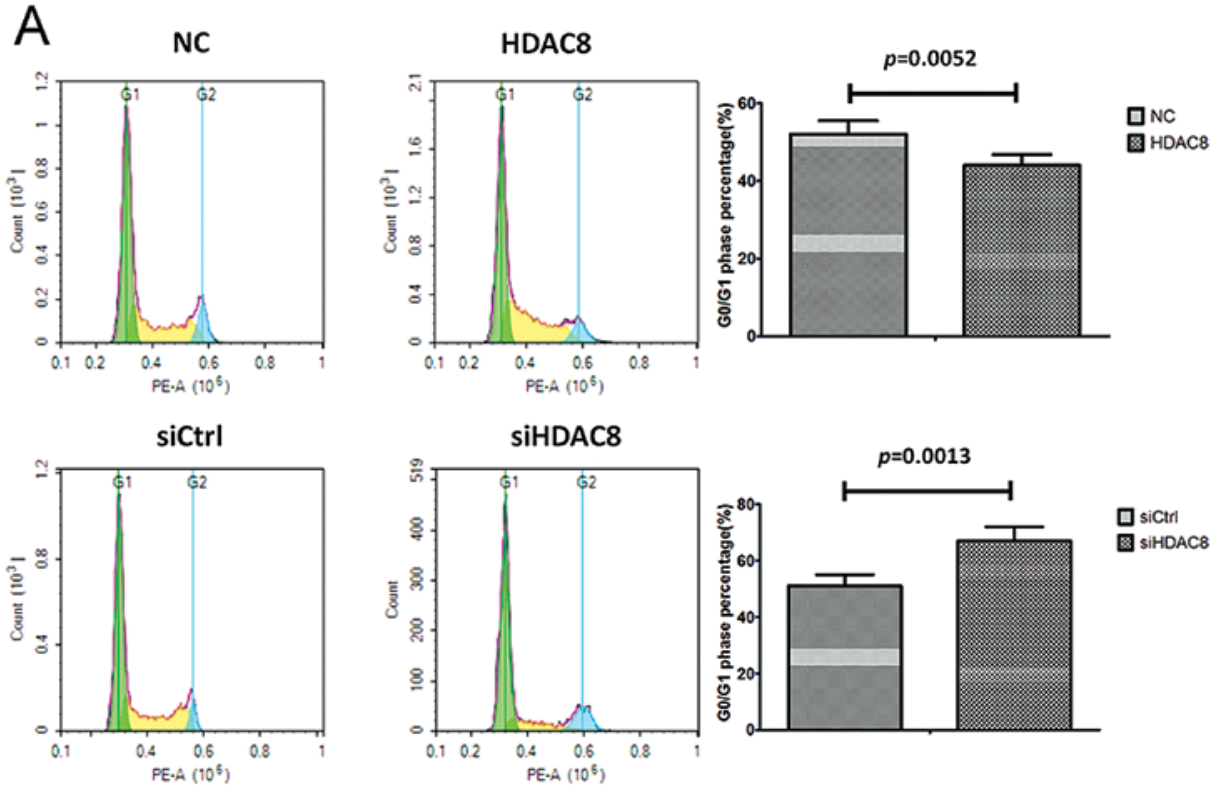

B

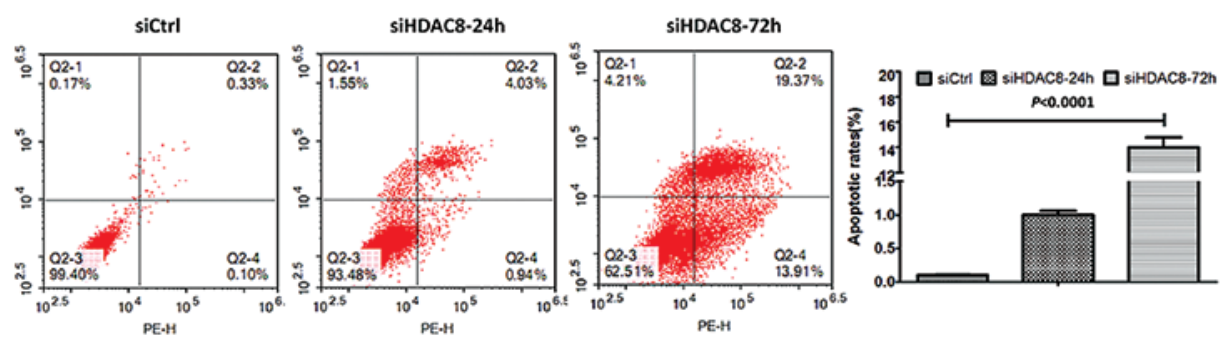

Figure 4. Depletion of HDAC8 significantly promoted G0/G1 arrest and apoptosis of the gastric cancer cell line MKN28. (A) G0/G1 phase percentage of examined MKN28 cells transfected with HDAC8 plasmid, siHDAC8 or controls after transfection was performed for 48 h. (B) Apoptosis rates of MKN28 cells treated with siHDAC8 or siCtrl at the designated time. Results in A and B are representative findings from three or more independent experiments, and all the values are shown as mean $\pm \mathrm{SEM}, \mathrm{n}=3$.

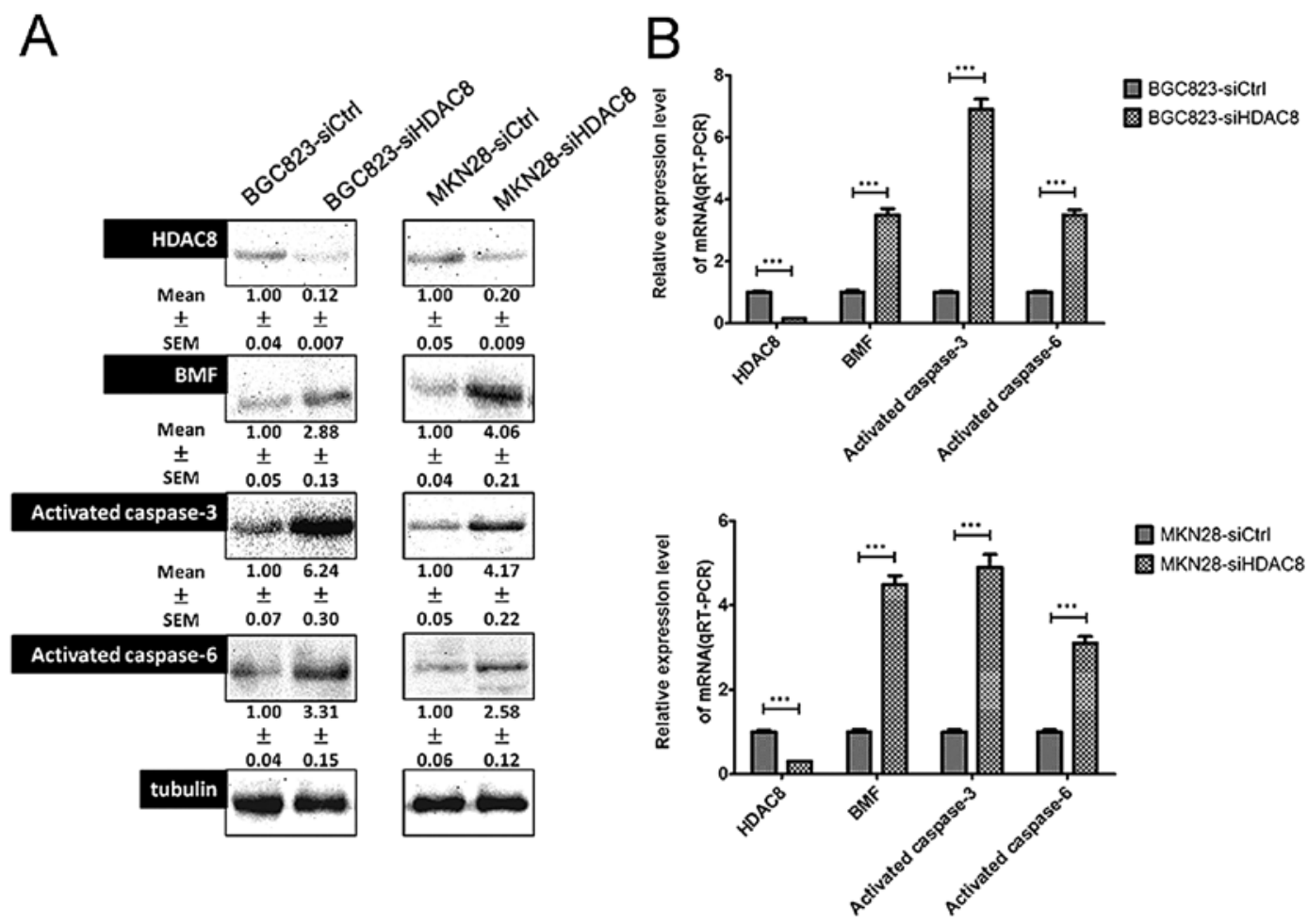

Figure 5. The effect of RNAi HDAC8 on the expression of related downstream proteins causing apoptosis. (A) Western blotting assay and (B) mRNA examined by quantitative RT-PCR (qRT-PCR) of BMF, activated caspase-3, activated caspase-6. Results in A are representative findings from three or more independent experiments, and all the values are shown as mean \pm SEM, $n=3$, tubulin served as a loading control $\left({ }^{* * *} \mathrm{P}<0.0001\right)$. 


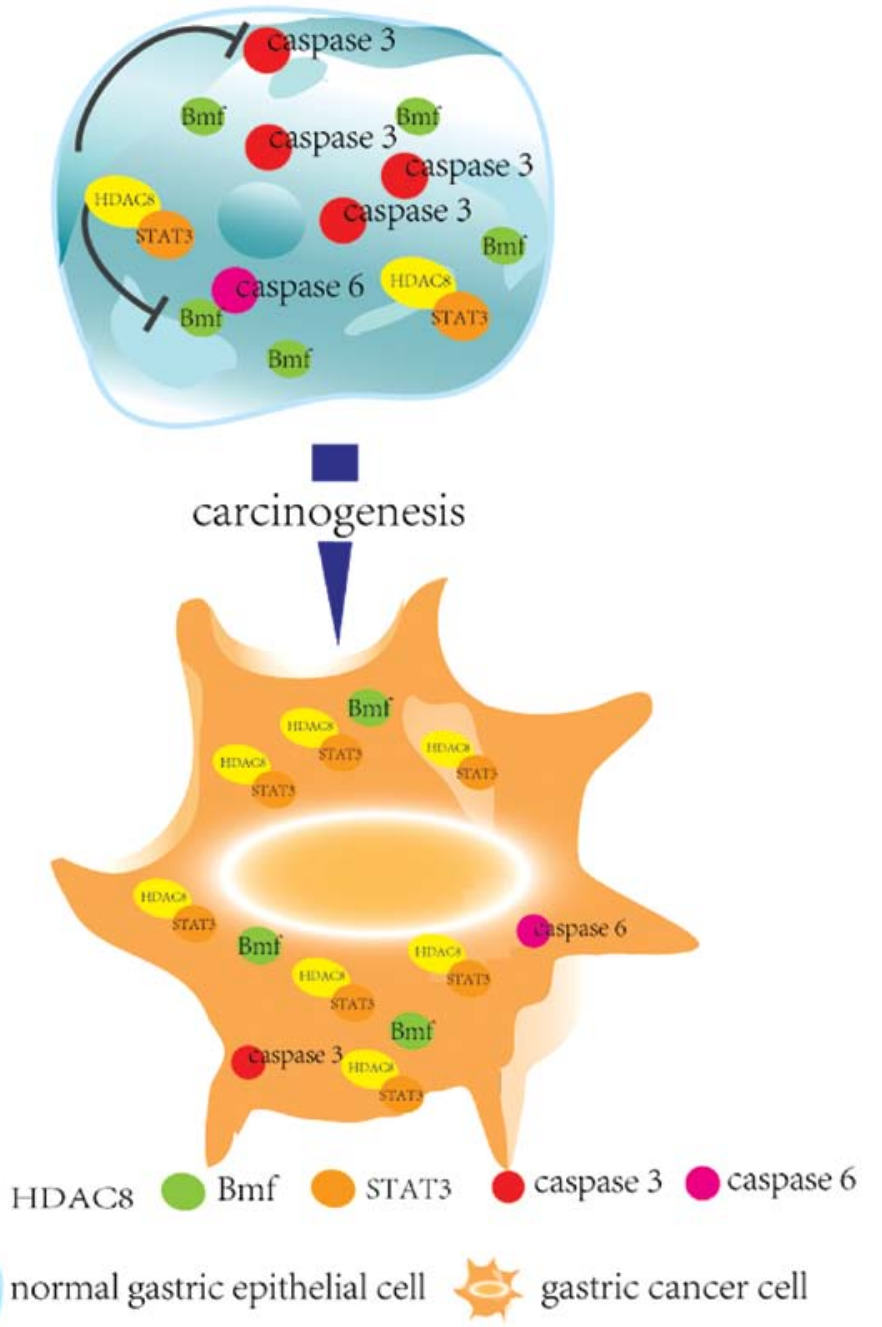

Figure 6. Schematic diagram of the effect of HDAC8 suppression in the growth of gastric cancer cells.

histone proteins modify the chromatin structure and form the basis for 'histone code'. The level of acetylation of histones and then the gene expression is controlled by the enzymes histone acetyl transferase (HAT) and histone deacetylase (HDAC). It was shown that the balance between HAT and HDAC was altered in many cancers (35).

HDACs are widely involved in cellular processes, ranging from cell differentiation to proliferation, senescence, and apoptosis; in particular, protecting a telomerase activator from ubiquitin-mediated degradation. Studies have shown that HDAC8 could serve as the prognostic biomarker, promote tumorigenesis and progress in multiple tumors. For example, Wilmott et al (36) found that HDAC8 may be a prognostic biomarker in melanoma, and also provide important data regarding the regulation of HDACs in melanoma and a rational basis for targeting them therapeutically; Wu et al (22) reported that HDAC8 was overexpressed in HCC and HDAC8 knockdown could suppress tumor growth and enhance apoptosis in HCC via elevating the expression of p53 and acetylation of p53 at Lys382, indicating that HDAC8 might serve as a potential therapeutic target in HCC (37); Oehme et al (16) found that the knockdown of HDAC8 resulted in the inhibition of proliferation, reduced clonogenic growth, cell cycle arrest and differentiation in cultured neuroblastoma cells; Balasubramanian et al (38) reported that HDAC8-selective inhibitors had a unique mechanism of action involving PLC $\gamma 1$ activation and calcium-induced apoptosis, and could offer benefits including a greater therapeutic index for treating T-cell malignancies. In the present study, we also found that depletion of HDAC8 using small interference RNA promoted apoptosis and cell cycle arrest in gastric cancer cells, moreover, forced expression of HDAC8 inhibited cell apoptosis and promoted cell proliferation, which suggested that HDAC8 may be a potential therapeutic target of gastric cancer.

$\mathrm{Bmf}, \mathrm{Bcl}-2$ modifying factor, is the closest relative of Bim (Bcl-2 interacting mediator of cell death) and functions as a tumor suppressor. A number of groups have demonstrated that overexpression of prosurvival Bcl-2 family members significantly reduces HDACi-mediated tumor cell death and therapeutic efficacy in preclinical models. In many cases, HDACi activate the intrinsic pathway via upregulation of a number of proapoptotic BH3-only Bcl-2 family genes including Bim, Bid and Bmf (39). Loss of bmf has been shown to accelerate the development of thymic lymphoma in a $\gamma$-irradiation carcinogenesis protocol in mice (40); BMF 
gene silencing in HT29 cells lead to a decrease in oxaliplatininduced cell death (41); Graab et al (42) found that GLI1/2 inhibitor GANT61 and PI3K/mTOR inhibitor PI103 cotreatment could increase mRNA and protein expression of NOXA and $\mathrm{BMF}$, which is required for apoptosis, since knockdown of NOXA or BMF significantly reduces GANT61/PI103induced apoptosis. It has been reported that HDAC8 mediated regulation of $\mathrm{Bcl}-2$-modifying factor $(\mathrm{BMF})$ via cooperation with STAT3. Here, we reported that inhibition of HDAC8 led to increased apoptosis rate of gastric cancer cells companied by the enhanced expression of Bmf, activated caspase- 3 and activated caspase-6 (Fig. 6).

In summary, in the present study, we demonstrated that HDAC8 was significantly upregulated in GC tissues and gastric cancer cells, and inhibition of HDAC8 inhibited cell proliferation and enhanced apoptosis. Moreover, we found that depletion of HDAC8 enhanced expression of Bmf, which is a tumor suppressor via inducing apoptosis. Our novel evaluation of HDAC8 in gastric adenocarcinoma may suggest new effective therapeutic strategies in GC.

\section{Acknowledgements}

The present study was supported by the National Natural Science Foundation of China (no. 81301763 and no. 81572849) and the Henan Provincial Key Scientific and Technological Projects (no. 142102310473).

\section{References}

1. Chen W, Zheng R, Zeng H, Zhang S and He J: Annual report on status of cancer in China, 2011. Chin J Cancer Res 27: 2-12, 2015.

2. Siegel RL, Miller KD and Jemal A: Cancer statistics, 2015. CA Cancer J Clin 65: 5-29, 2015

3. de Ruijter AJ, van Gennip AH, Caron HN, Kemp S and van Kuilenburg AB: Histone deacetylases (HDACs): Characterization of the classical HDAC family. Biochem J 370: 737-749, 2003.

4. Witt O, Deubzer HE, Milde T and Oehme I: HDAC family: What are the cancer relevant targets? Cancer Lett 277: 8-21, 2009.

5. Hait NC, Avni D, Yamada A, Nagahashi M, Aoyagi T, Aoki H, Dumur CI, Zelenko Z, Gallagher EJ, Leroith D, et al: The phosphorylated prodrug FTY720 is a histone deacetylase inhibitor that reactivates ER $\alpha$ expression and enhances hormonal therapy for breast cancer. Oncogenesis 4: e156, 2015.

6. Seicean A, Petrusel L, Seicean R, To C, Seicean A and Street C: New targeted therapies in pancreatic cancer. World J Gastroenterol 21: 6127-6145, 2015.

7. Eigl BJ, North S, Winquist E, Finch D, Wood L, Sridhar SS, Powers J, Good J, Sharma M, Squire JA, et al: A phase II study of the HDAC inhibitor SB939 in patients with castration resistant prostate cancer: NCIC clinical trials group study IND195. Invest New Drugs 33: 969-976, 2015.

8. Yang L, Liang Q, Shen K, Ma L, An N, Deng W, Fei Z and Liu J: A novel class I histone deacetylase inhibitor, I-7ab, induces apoptosis and arrests cell cycle progression in human colorectal cancer cells. Biomed Pharmacother 71: 70-78, 2015.

9. Bishayee K, Khuda-Bukhsh AR and Huh SO: PLGA-loaded gold-nanoparticles precipitated with quercetin downregulate HDAC-Akt activities controlling proliferation and activate p53-ROS crosstalk to induce apoptosis in hepatocarcinoma cells. Mol Cells 38: 518-527, 2015.

10. Pai JT, Hsu CY, Hua KT, Yu SY, Huang CY, Chen CN, Liao CH and Weng MS: NBM-T-BBX-OS01, semisynthesized from osthole, induced G1 growth arrest through HDAC6 inhibition in lung cancer cells. Molecules 20: 8000-8019, 2015.
11. Bian J, Zhang L, Han Y, Wang C and Zhang L: Histone deacetylase inhibitors: Potent anti-leukemic agents. Curr Med Chem 22: 2065-2074, 2015.

12. Zhu L, Yang J, Zhao L, Yu X, Wang L, Wang F, Cai Y and Jin J: Expression of hMOF, but not HDAC4, is responsible for the global histone H4K16 acetylation in gastric carcinoma. Int J Oncol 46: 2535-2545, 2015.

13. Dali-Youcef N, Froelich S, Moussallieh F-M, Chibbaro S, Noël G, Namer IJ, Heikkinen S and Auwerx J: Gene expression mapping of histone deacetylases and co-factors, and correlation with survival time and 1H-HRMAS metabolomic profile in human gliomas. Sci Rep 5: 9087, 2015.

14. Khan MA, Hussain A, Sundaram MK, Alalami U, Gunasekera D, Ramesh L, Hamza A and Quraishi U: (-)-Epigallocatechin-3gallate reverses the expression of various tumor-suppressor genes by inhibiting DNA methyltransferases and histone deacetylases in human cervical cancer cells. Oncol Rep 33: 1976-1984, 2015.

15. Gregoretti IV, Lee YM and Goodson HV: Molecular evolution of the histone deacetylase family: Functional implications of phylogenetic analysis. J Mol Biol 338: 17-31, 2004.

16. Oehme I, Deubzer HE, Wegener D, Pickert D, Linke JP, Hero B, Kopp-Schneider A, Westermann F, Ulrich SM, von Deimling A, et al: Histone deacetylase 8 in neuroblastoma tumorigenesis. Clin Cancer Res 15: 91-99, 2009.

17. Marks P, Rifkind RA, Richon VM, Breslow R, Miller T and Kelly WK: Histone deacetylases and cancer: Causes and therapies. Nat Rev Cancer 1: 194-202, 2001.

18. Pandey R, Müller A, Napoli CA, Selinger DA, Pikaard CS, Richards EJ, Bender J, Mount DW and Jorgensen RA: Analysis of histone acetyltransferase and histone deacetylase families of Arabidopsis thaliana suggests functional diversification of chromatin modification among multicellular eukaryotes. Nucleic Acids Res 30: 5036-5055, 2002.

19. Falkenberg KJ and Johnstone RW: Histone deacetylases and their inhibitors in cancer, neurological diseases and immune disorders. Nat Rev Drug Discov 13: 673-691, 2014.

20. Mottamal M, Zheng S, Huang TL and Wang G: Histone deacetylase inhibitors in clinical studies as templates for new anticancer agents. Molecules 20: 3898-3941, 2015.

21. Chakrabarti A, Oehme I, Witt O, Oliveira G, Sippl W, Romier C, Pierce RJ and Jung M: HDAC8: A multifaceted target for therapeutic interventions. Trends Pharmacol Sci 36: 481-492, 2015.

22. Wu J, Du C, Lv Z, Ding C, Cheng J, Xie H, Zhou L and Zheng S: The up-regulation of histone deacetylase 8 promotes proliferation and inhibits apoptosis in hepatocellular carcinoma. Dig Dis Sci 58: 3545-3553, 2013.

23. Vannini A, Volpari C, Filocamo G, Casavola EC, Brunetti M, Renzoni D, Chakravarty P,Paolini C, De Francesco R, Gallinari P, et al: Crystal structure of a eukaryotic zinc-dependent histone deacetylase, human HDAC8, complexed with a hydroxamic acid inhibitor. Proc Natl Acad Sci USA 101: 15064-15069, 2004

24. Kang Y, Nian H, Rajendran P, Kim E, Dashwood WM, Pinto JT, Boardman LA, Thibodeau SN, Limburg PJ, Löhr CV, et al: HDAC8 and STAT3 repress BMF gene activity in colon cancer cells. Cell Death Dis 5: e1476, 2014.

25. Nian H, Bisson WH, Dashwood WM, Pinto JT and Dashwood RH: $\alpha$-keto acid metabolites of organoselenium compounds inhibit histone deacetylase activity in human colon cancer cells. Carcinogenesis 30: 1416-1423, 2009.

26. Sargent JM and Taylor CG: Appraisal of the MTT assay as a rapid test of chemosensitivity in acute myeloid leukaemia. Br J Cancer 60: 206-210, 1989.

27. van de Loosdrecht AA, Beelen RH, Ossenkoppele GJ, Broekhoven MG and Langenhuijsen MM: A tetrazolium-based colorimetric MTT assay to quantitate human monocyte mediated cytotoxicity against leukemic cells from cell lines and patients with acute myeloid leukemia. J Immunol Methods 174: 311-320, 1994.

28. Twentyman PR and Luscombe M: A study of some variables in a tetrazolium dye (MTT) based assay for cell growth and chemosensitivity. Br J Cancer 56: 279-285, 1987.

29. Tolosa L, Donato MT and Gómez-Lechón MJ: General cytotoxicity assessment by means of the MTT assay. Methods Mol Biol 1250: 333-348, 2015.

30. Wang Y, Zhang D, Wu K, Zhao Q, Nie Y and Fan D: Long noncoding RNA MRUL promotes ABCB1 expression in multidrug-resistant gastric cancer cell sublines. Mol Cell Biol 34: 3182-3193, 2014. 
31. Guzmán C, Bagga M, Kaur A, Westermarck J and Abankwa D: ColonyArea: An ImageJ plugin to automatically quantify colony formation in clonogenic assays. PLoS One 9: e92444, 2014.

32. Feng X, Wang Y, Ma Z, Yang R, Liang S, Zhang M, Song S, Li S, Liu G, Fan D, et al: MicroRNA-645, up-regulated in human adencarcinoma of gastric esophageal junction, inhibits apoptosis by targeting tumor suppressor IFIT2. BMC Cancer 14: 633, 2014

33. Kennecke H, Yerushalmi R, Woods R, Cheang MC, Voduc D, Speers $\mathrm{CH}$, Nielsen TO and Gelmon K: Metastatic behavior of breast cancer subtypes. J Clin Oncol 28: 3271-3277, 2010.

34. Lassmann S, Shen Y, Jütting U, Wiehle P, Walch A, Gitsch G, Hasenburg A and Werner M: Predictive value of Aurora-A/ STK15 expression for late stage epithelial ovarian cancer patients treated by adjuvant chemotherapy. Clin Cancer Res 13: 4083-4091, 2007.

35. Lakshmaiah KC, Jacob LA, Aparna S, Lokanatha D and Saldanha SC: Epigenetic therapy of cancer with histone deacetylase inhibitors. J Cancer Res Ther 10: 469-478, 2014.

36. Wilmott JS, Colebatch AJ, Kakavand H, Shang P, Carlino MS, Thompson JF, Long GV, Scolyer RA and Hersey P: Expression of the class 1 histone deacetylases HDAC 8 and 3 are associated with improved survival of patients with metastatic melanoma. Mod Pathol 28: 884-894, 2015.
37. Tian Y, To KF, Lai P, Cheung YS, Wong VWS, Chan HLY and Cheng ASL: Histone deacetylase 8 is a novel chromatin modulator in NAFLD-associated hepatocarcinogenesis. Clin Gastroenterol Hepatol 13: 219, 2015.

38. Balasubramanian S, Ramos J, Luo W, Sirisawad M, Verner E and Buggy JJ: A novel histone deacetylase 8 (HDAC8)-specific inhibitor PCI-34051 induces apoptosis in T-cell lymphomas. Leukemia 22: 1026-1034, 2008.

39. Matthews GM, Newbold A and Johnstone RW: Intrinsic and extrinsic apoptotic pathway signaling as determinants of histone deacetylase inhibitor antitumor activity. Adv Cancer Res 116: 165-197, 2012.

40. Labi V, Grespi F, Baumgartner F and Villunger A: Targeting the Bcl-2-regulated apoptosis pathway by BH3 mimetics: A breakthrough in anticancer therapy? Cell Death Differ 15: 977-987, 2008.

41. Ginés A, Bystrup S, Ruiz de Porras V, Guardia C, Musulén E, Martínez-Cardús A, Manzano JL, Layos L, Abad A and Martínez-Balibrea E: PKM2 subcellular localization is involved in oxaliplatin resistance acquisition in HT29 human colorectal cancer cell lines. PLoS One 10: e0123830, 2015.

42. Graab U, Hahn H and Fulda S: Identification of a novel synthetic lethality of combined inhibition of hedgehog and PI3K signaling in rhabdomyosarcoma. Oncotarget 6: 8722-8735, 2015. 